\title{
RESEARCH
}

Open Access

\section{Bacteria pathogens drive host colonic epithelial cell promoter hypermethylation of tumor suppressor genes in colorectal cancer}

Xiaoxuan $\mathrm{Xia}^{1 \dagger}{ }^{1 \dagger}$, William Ka Kei Wu ${ }^{2,3+}$, Sunny Hei Wong ${ }^{3,4 \dagger}$, Dabin Liu ${ }^{3,4}$, Thomas Ngai Yeung Kwong ${ }^{3,4}$, Geicho Nakatsu', ${ }^{3,4}$, Pearlly S. Yan ${ }^{5}$, Yu-Ming Chuang ${ }^{6}$, Michael Wing-Yan Chan', Olabisi Oluwabukola Coker ${ }^{3,4}$, Zigui Chen ${ }^{7}$, Yun Kit Yeoh7, Liuyang Zhao ${ }^{3,4}$, Xiansong Wang ${ }^{3,4}$, Wing Yin Cheng ${ }^{3,4}$, Matthew Tak Vai Chan², Paul Kay Sheung Chan ${ }^{7}$, Joseph Jao Yiu Sung ${ }^{3,4}$, Maggie Haitian Wang ${ }^{1 *}$ and Jun $\mathrm{Yu}^{3,4,8^{*}}$ (D)

\begin{abstract}
Background: Altered microbiome composition and aberrant promoter hypermethylation of tumor suppressor genes (TSGs) are two important hallmarks of colorectal cancer (CRC). Here we performed concurrent $16 \mathrm{~S}$ rRNA gene sequencing and methyl-CpG binding domain-based capture sequencing in 33 tissue biopsies (5 normal colonic mucosa tissues, 4 pairs of adenoma and adenoma-adjacent tissues, and 10 pairs of CRC and CRC-adjacent tissues) to identify significant associations between TSG promoter hypermethylation and CRC-associated bacteria, followed by functional validation of the methylation-associated bacteria.
\end{abstract}

Results: Fusobacterium nucleatum and Hungatella hathewayi were identified as the top two methylation-regulating bacteria. Targeted analysis on bona fide TSGs revealed that $H$. hathewayi and Streptococcus spp. significantly correlated with CDX2 and MLH1 promoter hypermethylation, respectively. Mechanistic validation with cell-line and animal models revealed that $F$. nucleatum and $H$. hathewayi upregulated DNA methyltransferase. $H$. hathewayi inoculation also promoted colonic epithelial cell proliferation in germ-free and conventional mice.

Conclusion: Our integrative analysis revealed previously unknown epigenetic regulation of TSGs in host cells through inducing DNA methyltransferase by F. nucleatum and H. hathewayi, and established the latter as CRCpromoting bacteria.

Keywords: Colorectal cancer, Microbiota, DNA methylation, MBDCap-Seq, DNMT

\footnotetext{
* Correspondence: maggiew@cuhk.edu.hk; junyu@cuhk.edu.hk

${ }^{+}$Xiaoxuan Xia, William Ka Kei Wu, Sunny Hei Wong are co-first authors.

'Division of Biostatistics, Centre for Clinical Research and Biostatistics, JC

School of Public Health and Primary Care, The Chinese University of Hong

Kong, Shatin, N.T., Hong Kong, Special Administrative Region of China

${ }^{3} \mathrm{Li}$ Ka Shing Institute of Health Sciences, The Chinese University of Hong

Kong, Shatin, N.T., Hong Kong, Special Administrative Region of China

Full list of author information is available at the end of the article
}

(c) The Author(s). 2020 Open Access This article is licensed under a Creative Commons Attribution 4.0 International License, which permits use, sharing, adaptation, distribution and reproduction in any medium or format, as long as you give appropriate credit to the original author(s) and the source, provide a link to the Creative Commons licence, and indicate if changes were made. The images or other third party material in this article are included in the article's Creative Commons licence, unless indicated otherwise in a credit line to the material. If material is not included in the article's Creative Commons licence and your intended use is not permitted by statutory regulation or exceeds the permitted use, you will need to obtain permission directly from the copyright holder. To view a copy of this licence, visit http://creativecommons.org/licenses/by/4.0/ The Creative Commons Public Domain Dedication waiver (http://creativecommons.org/publicdomain/zero/1.0/) applies to the data made available in this article, unless otherwise stated in a credit line to the data. 


\section{Introduction}

Colorectal cancer (CRC), the fourth most common cancer and the fifth leading cause of cancer death globally [1], occurs as a result of intricate interactions between host and environmental factors, among which gut dysbiosis is strongly associated with disease occurrence [2, 3]. In particular, metagenomic studies by us and other investigators have demonstrated compositional alterations of stool and mucosal microbiomes across stages of colorectal tumorigenesis that are characterized by ectopic overgrowth of oral pathogens and depletion of multiple commensal species [4-7]. Subsequent functional studies of CRC-enriched species have further led to identification of pivotal mutagenic or tumor-promoting bacteria (e.g., pks-positive Escherichia coli, Fusobacterium nucleatum, Peptostreptococcus anaerobius) that play direct causative roles in CRC [8-10]. Transplantation of stool from CRC patients into germ-free and conventional mice also confirmed the causal link of gut microbes to CRC development [11]. These evidences collectively support a key functional role of bacteria in CRC pathogenesis.

Both genetic and epigenetic pathways are involved in CRC development, in which the latter aberrantly silences tumor suppressor genes (TSGs) via multiple mechanisms, including promoter hypermethylation and histone modifications [12]. DNA methylation involves the covalent addition of a methyl group to the 5-carbon of the cytosine ring within CG dinucleotides of gene promoters by DNA methyltransferases (DNMTs) to form 5-methylcytosine (5-mC). In this regard, gene-specific promoter hypermethylation has been widely reported in CRC with many methylated genes functionally verified as bona fide TSGs (e.g., APC, MLH1, PTEN, RUNX3, CDX1/2) [13]. Nevertheless, the upstream molecular events regulating DNA methylation in relation to etiological factors of CRC remain largely elusive.

Sporadic studies have attempted to investigate the effects of the gut microbiome or specific microorganisms on DNA methylation in colonic epithelial cells. In a porcine model, early microbial colonization was found to affect DNA methylation of genes related to immune response, phagocytosis, endothelial homeostasis, and tissue metabolism [14]. Exposure to probiotics or pathogenic bacteria in the context of necrotizing enterocolitis that primarily affects preterm infants has also been shown to induce differential DNA methylation patterns in colonic epithelial cells [15]. Moreover, the gut microbiome was found to be essential for guiding epigenetic development of intestinal stem cells during early development [16]. These studies suggest the existence of an important linkage between the gut microbiome and the host-cell methylome. The microbiome-methylome axis in CRC, however, has not been systematically investigated. Here, we performed concurrent profiling of the methylome and mucosal microbiome in CRC patients to investigate associations between methylation signatures and microbial abundances. We demonstrated that CRC-associated bacteria modulated DNA methylation patterns in colonic epithelial cells to drive intestinal tumorigenesis.

\section{Methods}

Patient recruitment and informed consent

We enrolled individuals who had undergone standardized colonoscopic examinations at the Prince of Wales Hospital of the Chinese University of Hong Kong. Written informed consents were obtained from subjects or their authorized representatives. Eligibility criteria for colonoscopy included (1) age 50-70 years; (2) absence of existing or previous CRC symptoms, such as hematochezia, tarry stool, change in bowel habit in the past 4 weeks or a weight loss of $>5 \mathrm{~kg}$ in the past 6 months; and (3) not having received any CRC screening tests in the past 5 years. Biopsies were snap-frozen in cryovials immediately after polypectomy and stored at $-80^{\circ} \mathrm{C}$ until DNA extraction. Adjacent normal tissues were taken at least $4 \mathrm{~cm}$ away from lesions. Colorectal mucosae were obtained using cold biopsy forceps separately for lesions and lesionsadjacent tissues to avoid cross-contamination between samples.

\section{Methylated-DNA capture sequencing}

Genomic DNA samples of colonic mucosal biopsies were obtained with informed consent as previously described [5]. Methylated DNA was enriched using the MethylMiner $^{\mathrm{TM}}$ kit (cat. ME10025, Invitrogen, US) [17]. Briefly, DNA was sonicated and incubated at a ratio of 1 : 3.5 with human MBD2-biotin conjugated to Dynabeads M-280 Streptavidin. Methylated DNA was purified from magnetic bead complex by performing 3 washes with Bind/Wash Buffer to remove the uncaptured fraction of DNA. Captured DNA was eluted with $1 \mathrm{M} \mathrm{NaCl}$ and precipitated in ethanol. Single-end 50-bp Illumina libraries were prepared for enriched DNA samples, which were subjected to sequencing on the Illumina Genome Analyzer IIx according to manufacturer's protocol.

\section{MBDCap sequence quality control}

Raw sequences were quality-trimmed using Trimmomatic (v0.36) in maximal information mode [18], which was configured at $99.9 \%$ strictness to find and trim any bases having Phred quality scores $<30$. Sequence reads were simultaneously screened, flagged for, and trimmed of Illumina adapter sequences; those that had fewer than 35 bases were finally discarded prior to downstream processing. We decontaminated post-Trimmomatic sequences using the Burrows-Wheeler Aligner with default parameters of maximal exact match algorithm (bwa-mem, v0.7.17-r1188) by aligning them against an indexed 
database consisting of 6877 bacterial plasmids, complete genomes of 8117 mitochondria, and 2116 plastomes as well as $4522 \mathrm{EmVec}$ and $6093 \mathrm{UniVec}$ sequencing vectors (NCBI RefSeq accessed on May 12, 2016) [19]. Sequences were mapped to dust-masked GENCODE human genome (GRCh38.p12) with bwa-mem aligner for detection and removal of optical and PCR duplicates using the MarkDuplicates module in the Picard toolkit (v2.14.0) [20].

\section{Statistical inference and analysis of genome-wide DNA methylome}

MBDCap sequence enrichment signals were modeled and transformed to infer absolute levels of DNA methylation using the QSEA R/Bioconductor package for which we performed parametrization and provided external calibration datasets [21]. Average single-end sequencing fragment length was estimated using the makeTagDirectory command in the HOMER bioinformatic suite [22]. An optimal size of sliding window for aligned reference coordinates in each sample BED file was selected by defining the lowest Akaike's information criterion (AIC) of read count density function across an incremental range of genomic segment sizes [23]. A custom Biostrings-based genome data infrastructure was created for the GENCODE human genome assemblies using the BSgenome R/Bioconductor package and imported into qseaSet object. We defined copy number variations $(\mathrm{CNVs})$ across 1 megabase segment with the built-in HMMcopy function to adjust for effects on read count normalization as cancer samples were included in our dataset [24]. A genomic segment was considered CpGfree if the pattern density was lower than $0.001 \%$, which was used to offset background reads. To calibrate parameters of $\mathrm{CpG}$ enrichment function, we obtained publicly available Illumina Infinium Methylation 450K BeadChip data. This calibration data included methylation profiles of COAD and READ projects from the GDC Data Portal of the Cancer Genome Atlas (TCGA) as well as of independent projects of a similar study design (GSE48684 and GSE53051) from the Gene Expression Omnibus (GEO) [25, 26]. These datasets were preprocessed through the Minfi R/ Bioconductor package and normalized separately via the SWAN algorithm [27, 28]. Potential effects of technical variation (TCGA plate IDs) were removed before combining normalized datasets to correct for study batches using the ComBat algorithm as implemented in the sva R/Bioconductor package [29]. We filtered Infinium 450K CpG probes that had average beta scores of $<0.9$ and standard deviations of $>0.05$ for a tissue phenotype group [21]; those that met these criteria were assigned to chromosomal coordinates to calculate average beta score for each 200-bp genome bin across primary human genome assemblies [30]. As a result, we identified 55,341 genomic regions that had low variability in methylation over an integrated set of TCGAGEO samples for calibration of enrichment profiles. To enable comprehensive genomic feature-guided methylome profiling and analysis, we imported GENCODE gff3 annotation file and unmasked CpG island information from the UCSC Golden Path services as well as genomic coordinates of FANTOM5-CAGE enhancers and miRNA promoters [31, 32]. Differentially methylated regions (DMRs) between tissue phenotypes and between microbiome subtypes were detected using the limma R/Bioconductor package [33], and robust pairwise fold change (pfc) was determined by calculating the average of pfc values that fell within an interquartile range [34]. Stratified hierarchical clustering was performed according to the Ward's minimum-variance method using Euclidian distance-based sample dissimilarity matrix; methylotypes were defined by a consensus analysis employing 30 indices for cluster optimization as implemented in NbClust $\mathrm{R} / \mathrm{CRAN}$ package.

\section{RNA extraction and real-time PCR}

Total RNA was extracted from cells and tissues using TRIzol Reagent (Molecular Research Center Inc.). cDNA was synthesized from $1 \mu \mathrm{g}$ of total RNA using Transcriptor Reverse Transcriptase (Roche). Real-time PCR was performed using SYBR Green master mixture (Roche) on LightCycler 480 Instrument. Each sample was tested in triplicate. $\Delta \Delta \mathrm{C}_{\mathrm{T}}$ method was applied to determine the fold change in gene expression. $\Delta \mathrm{C}_{\mathrm{T}}$ method was applied to determine the relative expression of corresponding genes.

\section{Western blots}

Total protein was separated by sodium dodecyl sulfatepolyacrylamide gel electrophoresis (SDS-PAGE). The proteins in SDS-PAGE were transferred onto nitrocellulose membranes (GE Healthcare). The membrane was incubated with primary antibodies overnight at $4{ }^{\circ} \mathrm{C}$, and then with secondary antibody at room temperature for $1 \mathrm{~h}$. Proteins of interest were visualized using ECL Plus Western blotting Detection Reagents (GE Healthcare).

\section{Global DNA methylation (5-mC) level analysis}

Two hundred nanograms of genome DNA were harvested, and DNA methylation levels were detected by MethylFlash Global DNA Methylation (5-mC) ELISA Easy Kit (p-1030, EpiGentek) according to the manufacturer's instructions. All experiments were conducted three times in triplicate. Results are shown as mean \pm SEM.

\section{DNMT activity assay}

Twenty micrograms of nuclear proteins were harvested, and DNMT activities were detected by DNMT assay kits (ab113469 for DNMT1, ab113470 for DNMT3A, and ab113471 for DNMT3B, Abcam) according to the manufacturer's instructions. All experiments were conducted three times in triplicate. Results are shown as mean \pm SEM. 


\section{Animal experiments}

Conventional C57BL/6 mice were bred in the Laboratory Animal Services Centre at the Prince of Wales Hospital, The Chinese University of Hong Kong. Male C57BL/6 mice ( 6 weeks of age) were treated with a cocktail of broad-spectrum antibiotics, including ampicillin (0.2 g/ $\mathrm{L})$, metronidazole $(0.2 \mathrm{~g} / \mathrm{L})$, neomycin $(0.2 \mathrm{~g} / \mathrm{L})$, and vancomycin $(0.1 \mathrm{~g} / \mathrm{L})$, in their drinking water for 1 week, followed by a single intraperitoneal injection of azoxymethane (AOM, $10 \mathrm{mg} / \mathrm{kg}$ ). One-week post AOM injection, mice were gavaged with $1 \times 10^{8}$ c.f.u. of $H$. hathewayi every 2 days for 6 weeks. For germ-free mice experiments, KunMing mice were bred at the Department of Laboratory Animal Science at the Third Military Medical University in Chongqing, China. Adult germfree mice ( 8 weeks of age) were gavaged with $H$. hathewayi $1 \times 10^{8}$ c.f.u. of $H$. hathewayi every 2 days for 20 weeks. At the end of each experiment, mice were anaesthetized with isoflurane followed by cardiac puncture to collect arterial blood, and cervical dislocation before dissecting for tissue collection.

\section{Mouse tissue collection and histological evaluation}

After sacrifice, the whole colon of the mice was flushed with phosphate-buffered saline (PBS) and excised longitudinally. The colon was first examined for erosion and abnormality, and then divided into 3 sections (proximal, middle, and distal). Half of each colon section was fixed in $10 \%$ formalin, processed and paraffin-embedded for histologic examination. The remaining half of each colon section was snap-frozen and kept at $-80^{\circ} \mathrm{C}$ for subsequent molecular analyses. Four $5-\mu \mathrm{m}$ tissue sections from each colonic segment were stained with hematoxylin and eosin. The Ki-67 expression of the paraffin-embedded tissues was analyzed by immunohistochemistry. The proportion of Ki-67 positive cells was determined by counting immunostain-positive cells, as a percentage to the total number of nuclei in the field.

\section{Statistical methods}

Differentiated methylation and microbiome segments were tested by the Wilcoxon signed rank test for paired samples, and the Mann-Whitney $U$ test for independent samples. Zero-inflated negative binomial regression was used to test for the interaction between the microbiome species count and methylation gene expression, implemented by $\mathrm{R}$ package pscl. The analysis was repeated using an alternative Spearman's rank-order correlation test to check for consistency. Heat maps were used to visualize differential abundances in the methylome and microbiome across different CRC phenotypes. All analyses were conducted in $\mathrm{R}$.

\section{Results}

Parallel methylome and microbiome profiling in colonic adenomas, $\mathrm{CRC}$, and corresponding adjacent tissues Genome-wide DNA methylation profiling by methyl-CpG binding domain-based capture sequencing (MBDCap-Seq) was performed in thirty-three tissue biopsies from five normal colonic mucosa tissues, four pairs of adenoma and adenoma-adjacent tissues, and ten pairs of CRC and CRCadjacent tissues. Genome-wide coverage of MBDCap sequence reads (Figure S1) and genome-wide distribution of differentially methylated regions across phenotypes (Figure S2) were first determined followed by targeted analysis of promoters of known TSGs (as curated by the TSGene database) [35]. Among the differentially methylated segments $(p<0.005$, Wilcoxon signed rank test for paired sample or Mann-Whitney $U$ test for unpaired sample comparison), the majority were hypermethylated in tumor samples as compared to corresponding adjacent controls. Moreover, one fifth of the $\mathrm{CpG}$ sites showed a progressive trend of methylation alterations along the adenomacarcinoma sequence (Fig. 1).

Parallel microbiome profiling from the same set of samples was performed by $16 \mathrm{~S}$ rRNA gene amplicon sequencing study (Figure S3) [5]. Original abundances of taxa were used to perform subsequent analysis as recommended by Weiss et al. [36]. Paired analysis of adenoma, CRC, and their adjacent tissues showed that F. nucleatum (fold change $=22.34, p=0.009$ ), Hungatella hathewayi (fold change $=9.67, p=0.042$ ), and Parvimonas spp. (fold change $=5.78, p=0.009$ ) were the top three bacteria that showed significant enrichment in tumor tissues compared to corresponding adjacent controls.

\section{F. nucleatum and $H$. hathewayi showed significantly stronger interactions with methylation of TSG promoters}

Next, we evaluated whether the abundances of mucosal microorganisms identified above were associated with differentially methylated TSGs. F. nucleatum and $H$. hathewayi had the highest number of significant associations with TSG promoter methylation across stages of CRC development ( $p<0.001$, zero-inflated negative binomial regression) (Fig. 2a). Furthermore, the interactions involving these two bacteria were predominantly positive in direction, with higher abundances correlating with higher levels of TSG promoter methylation. $H$. hathewayi was associated with methylation of TSGs including SOX11, THBD, SFRP2, GATA5, and ESR1, whereas $F$. nucleatum was associated with methylation of MTSS1, RBM38, PKD1, and PTPRT (Fig. 2b; Table $\mathrm{S} 1$ ). We also observed that, while associations between bacteria and TSG promoter methylation status were composed of a mixture of positive and negative correlations in the normal colonic mucosa samples, the direction of correlations was uniformly positive in CRC 


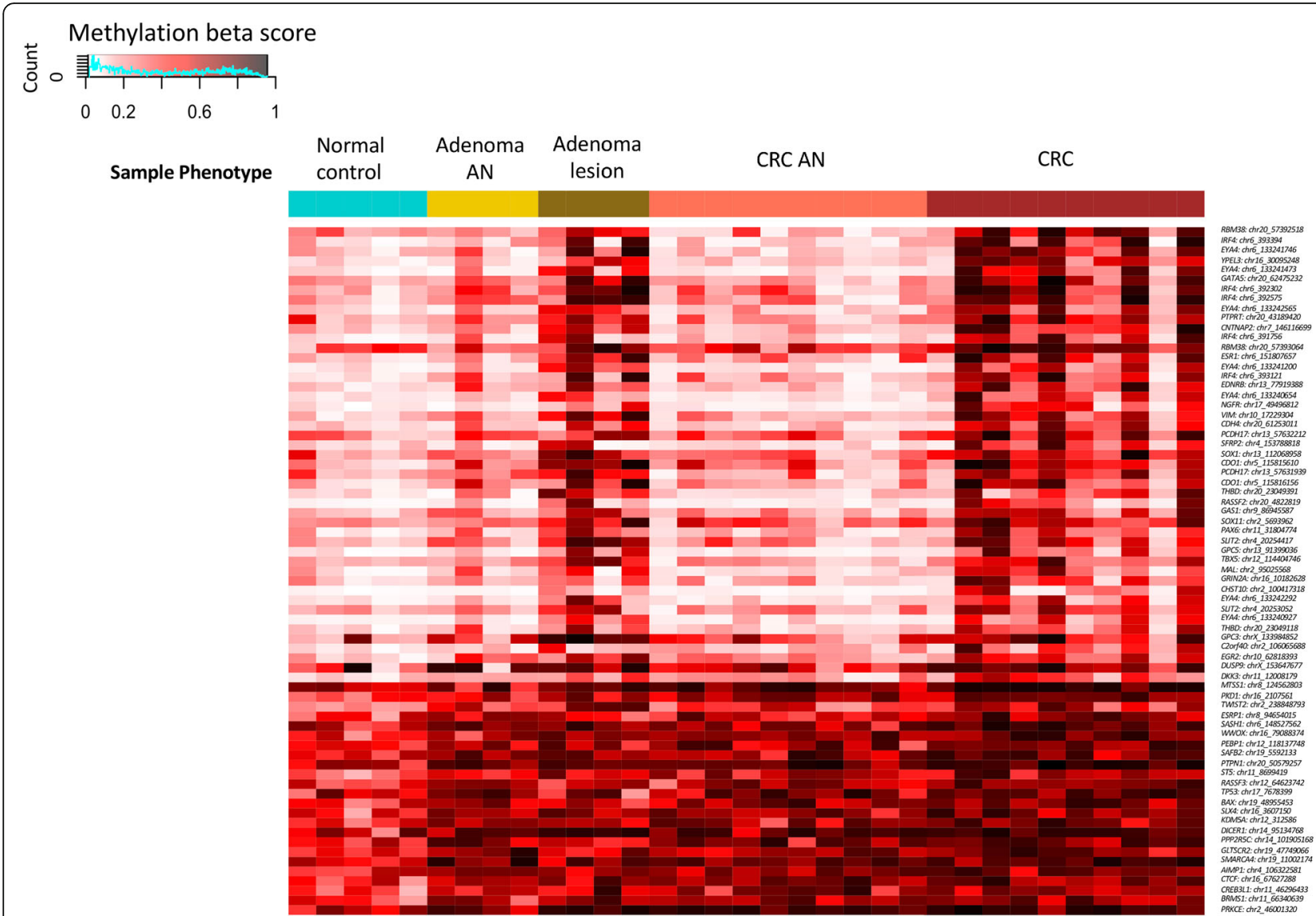

Fig. 1 Tumor suppressor genes (TSGs) hypermethylation in adenomas and CRC. Heatmap of TSG promoter regions showed pervasive hypermethylation in adenomas and CRC samples. The methylation segments shown are the genes with differential methylation between any two phenotype groups $(P<0.005)$. AN, adjacent

samples (Fig. 2c). These results suggested that enrichment of $H$. hathewayi and $F$. nucleatum were associated with pervasive TSG-promoter methylation. Statistical replication was performed using an alternative non-parametric test by the Spearman rank correlation. Consistent with the results from zero-inflated negative binomial regression, EYA4 promoter-wide methylation was positively associated with both $H$. hathewayi (negative binomial $p=2.87 \mathrm{E}-15$, Spearman $p=2.21 \mathrm{E}-4$ ) and $F$. nucleatum (negative binomial $p=$ 1.65E-14, Spearman $p=2.15 \mathrm{E}-12$ ) as displayed in the violin plots (Fig. 2d).

\section{Bacteria interactions with bona fide TSGs in CRC}

MLH1, APC, PTEN, P16, and CDX1/2 are bona fide TSGs whose promoter hypermethylation and tumorsuppressing functions in CRC have been experimentally verified [13]. To investigate whether the mucosal microbiome is associated with methylation profiles of these specific TSGs, we evaluated all bacteria for interactions with these key TSGs. In this regard, MLH1, APC, PTEN, and CDX2 showed positive interactions with multiple CRC-enriched bacteria (Fig. 3). Particularly, strong positive correlations between methylation of multiple $\mathrm{CpG}$ sites in the promoter of $C D X 2$ and $H$. hathewayi, as well as $M L H 1$ and Streptococcus spp. were observed. Hypermethylation of the promoter of $A P C$ was also positively associated with $H$. hathewayi and Streptococcus spp. (Table S2). These results suggested that $\mathrm{CRC}$-enriched bacteria might be a potential driver of hypermethylation in the promoter regions of key TSGs in CRC.

\section{F. nucleatum and $H$. hathewayi increased DNMT expression and activity in colonic cell lines}

Next, we investigated the downstream molecular mechanisms underlying the potential hypermethylation effects of $H$. hathewayi and $F$. nucleatum in CRC. Because DNMTs are a family of enzymes that initiate CpG methylation, we postulated that $F$. nucleatum and $H$. hathewayi might cause TSG promoter hypermethylation by regulating DNMT expression and activity. To confirm this, a colonic normal epithelial cell line (NCM460) and two colon CRC cell 


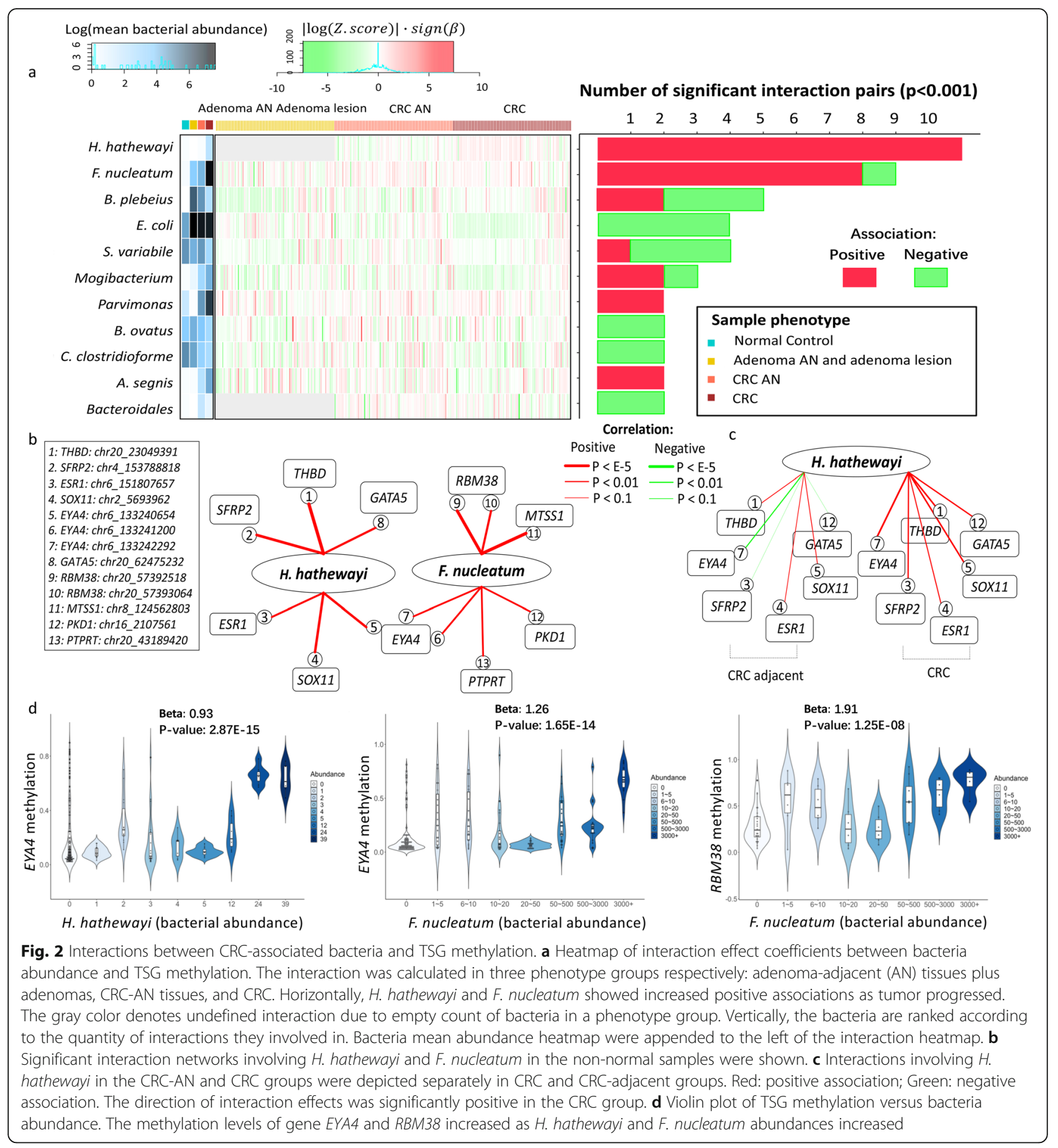

lines (HT29 and HCT116) were incubated with F. nucleatum and $H$. hathewayi for $4 \mathrm{~h}$. We found that incubation with $F$. nucleatum and $H$. hathewayi led to significant increases in global DNA methylation (5-mC) levels in NCM460 ( $p<0.05$ for H. hathewayi and $p<0.05$ for $F$. nucleatum), HCT116 ( $p<0.05$ for $H$. hathewayi and $p<$ 0.01 for F. nucleatum) and HT29 ( $p<0.01$ for $H$. hathewayi and $p<0.05$ for $F$. nucleatum) compared to PBS treatment or incubation with non-tumorigenic
Escherichia coli MG1655 (Fig. 4a). Consistent with these observations, $F$. nucleatum and $H$. hathewayi induced the expression and nuclear activity of DNMT1 and DNMT3A in two CRC cell lines (HT29 and HCT116) (Fig. 4b-d). In the normal epithelial cell line NCM460, $H$. hathewayi also increased the expression and nuclear activity of DNMT1 and DNMT3A, whereas F. nucleatum induced mRNA expression and nuclear activity of DNMT3B (Fig. 4c and d). 
1: MLH1:chr3_36993412

2: MLH1: chr3 36994231

3: $M L H 1:$ chr3_36995323

4: MLH1: chr3_37009792

5: MLH1: chr3 37010065

6: MLH1: chr3_37012795

7: MLH1:chr3_37026445

8: MLH1: chr3 37026718

9: APC: chr5 112707232

10: APC: chr5_112736443

11: APC: chr5 112737808

12: APC: chr5_112738081

13: PTEN: chr10_87862867

14: PTEN: chr10 87878155

15: PTEN: chr10_87953776

16: CDX2: chr13_27969943

17: CDX2: chr13_27970216

18: CDX2: chr13_27970489

19: CDX2: chr13_27970762

20: CDX2: chr13_27971035

b

Beta $=1.17$

P-value $=0.0013$

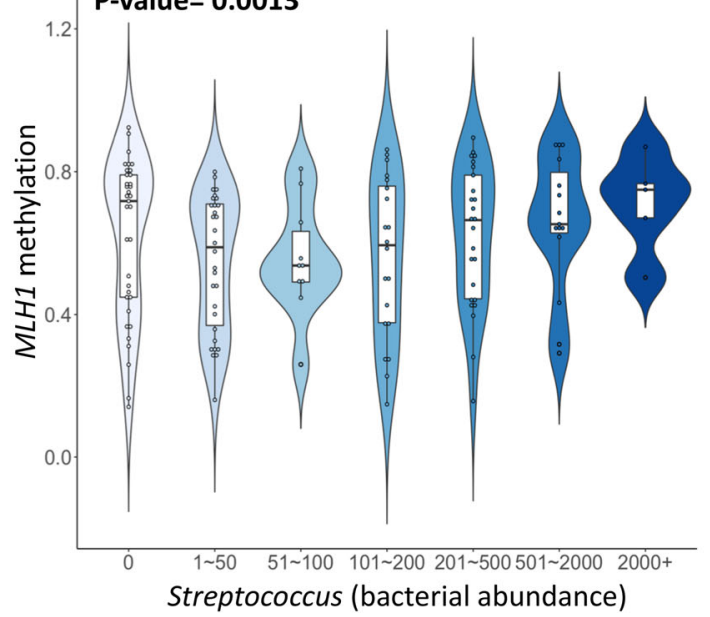

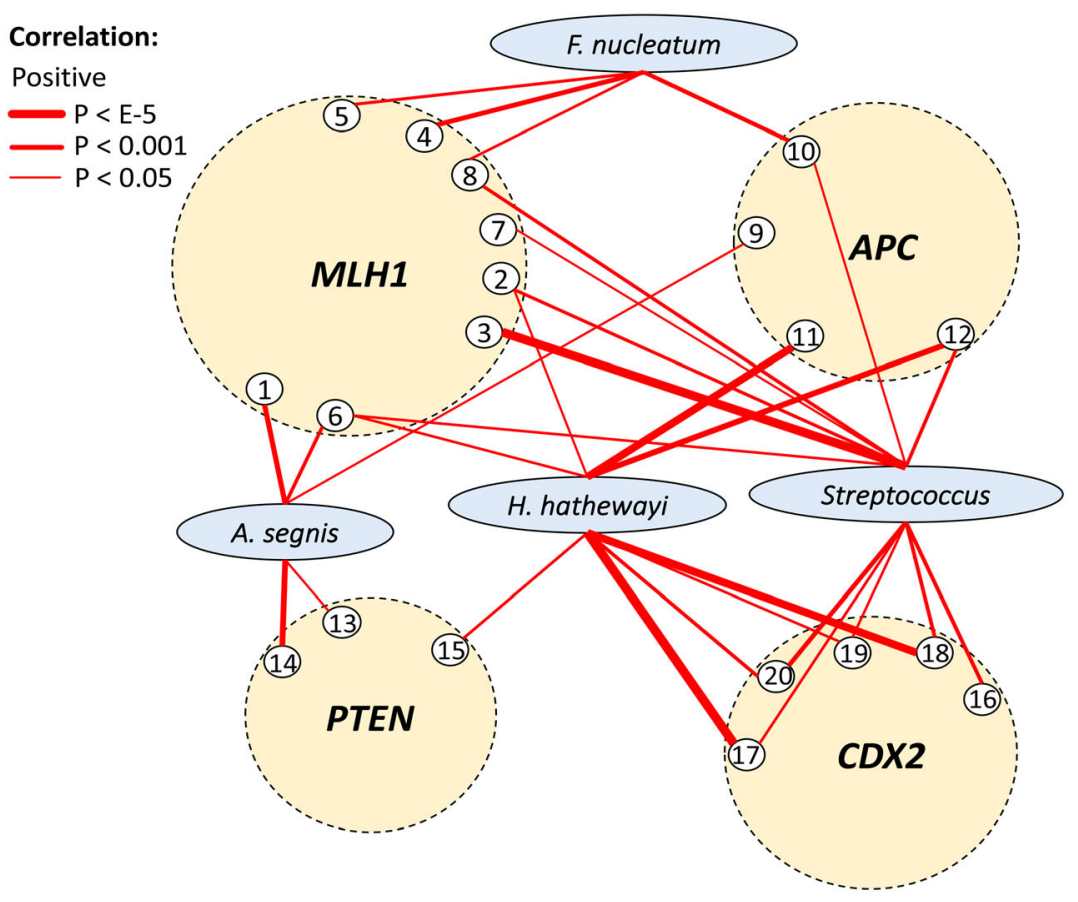

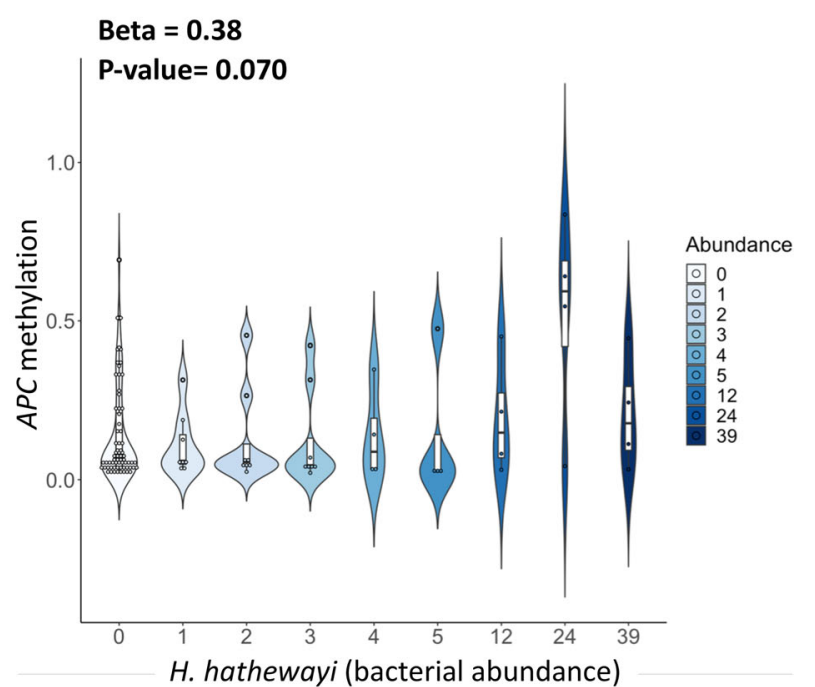

Fig. 3 Bacteria interactions with target CRC TSGs. a Significant positive bacteria interactions involving MLH1, APC, PTEN, P16, CDX1, and CDX2. Line width $=-\log (P)$. $\mathbf{b}$ Left: Violin plot of MLH1 methylation versus Streptococcus spp. Right: APC versus H. hathewayi. Methylation levels of both genes increased as the bacteria abundance elevated

H. hathewayi promoted intestinal epithelial cell proliferation in conventional and germ-free mice and induced DNMT expression and activity in vivo

Our results indicated that upregulation of DNMT expression and activity was associated with F. nucleatumand $H$. hathewayi-induced TSG promoter hypermethylation. F. nucleatum has been well characterized as a CRC-promoting bacterium [37], whereas the role of $H$. hathewayi in intestinal tumorigenesis remains unknown. To assess the potential tumor-promoting activity of $H$. hathewayi, we examined its in vivo effects in two separate mouse models, using germ-free and conventional specific pathogen-free (SPF) mice. Germ-free mice gavaged with $H$. hathewayi showed a significant increase in colonic Ki-67 immunoreactivity, when compared to control mice given PBS ( $p<0.01$ ) (Fig. 5a). Consistently, in conventional microbiota-depleted mice (using antibiotics) challenged with azoxymethane (AOM; a colonspecific chemical carcinogen) followed by $H$. hathewayi or PBS gavage, we observed significantly increased cell 


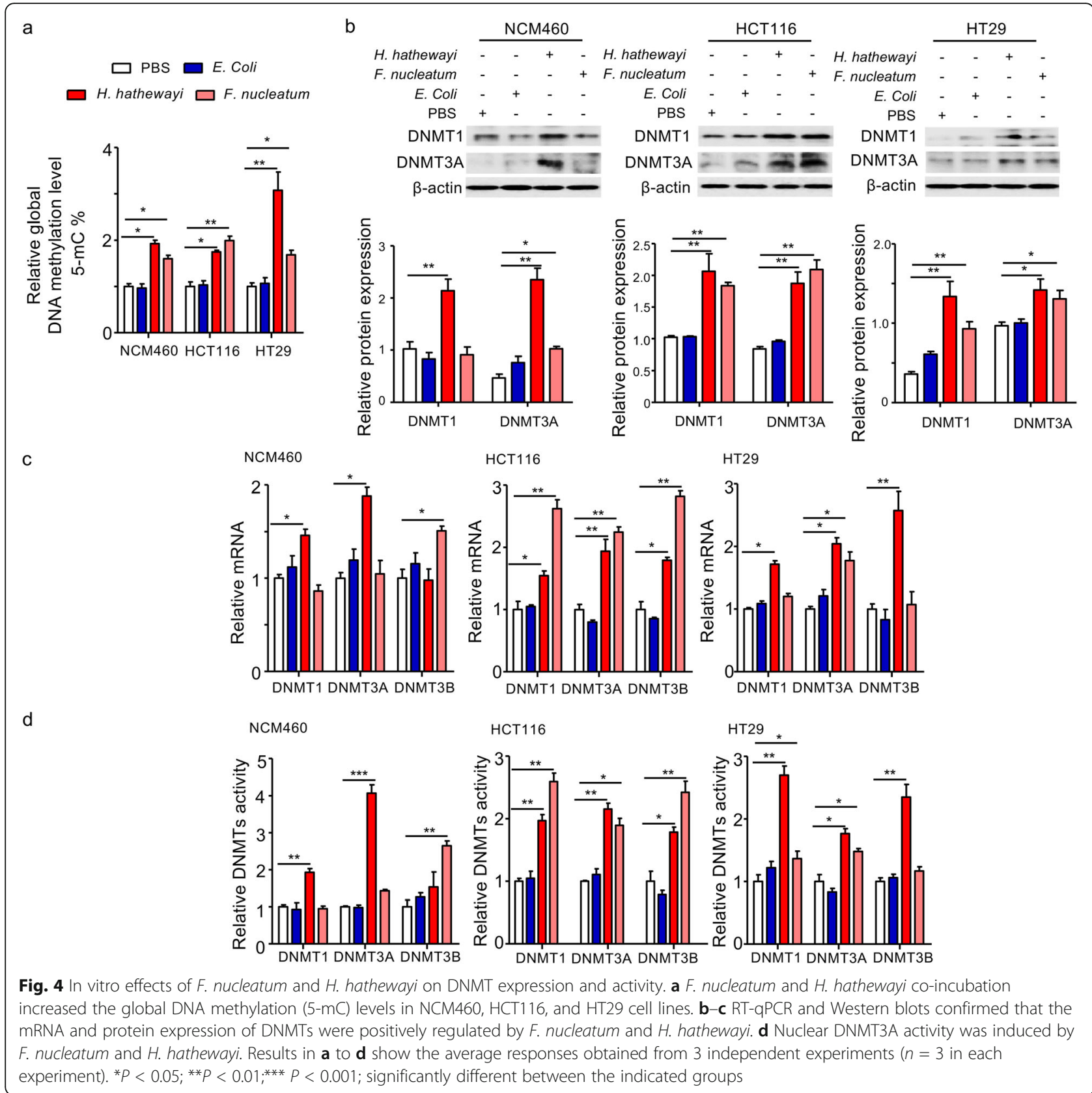

proliferation $(p<0.01)$ in the colon tissue of the $H$. hathewayi group as indicated by a higher proportion of Ki-67 positive cells (Fig. 5b).

In line with our in vitro findings, we observed an upregulation of DNMT1 and DNMT3A protein in $H$. hathewayi-inoculated conventional mice and germ-free mice as compared with corresponding untreated control mice (Fig. 5c and d). Meanwhile, we noted a similar effect in $F$. nucleatum-inoculated conventional mice (Fig. 5d) but not in F. nucleatum-inoculated germ-free mice (data not shown). These data collectively showed that enrichment of $F$. nucleatum or $H$. hathewayi in colonic mucosa activates DNMT expression and activity. Taken together, these results indicate that $H$. hathewayi could promote intestinal cell proliferation at least in part by modulating host methylation through inducing DNMT. Aside from DNMT, we observed that $H$. hathewayi could induce the mRNA expression of prostaglandin $E_{2}$ biosynthetic pathway genes Pla2g4c and Cox2 (Figure S4).

\section{Discussion}

In the present study, through concurrent MBDCap-Seq and 16S rRNA gene sequencing from the same biopsies, we discovered novel associations between tumoral 

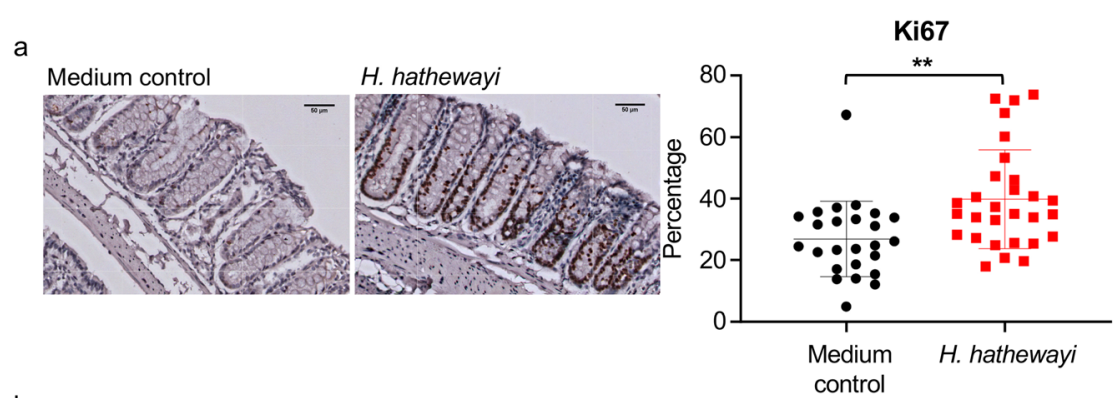

b
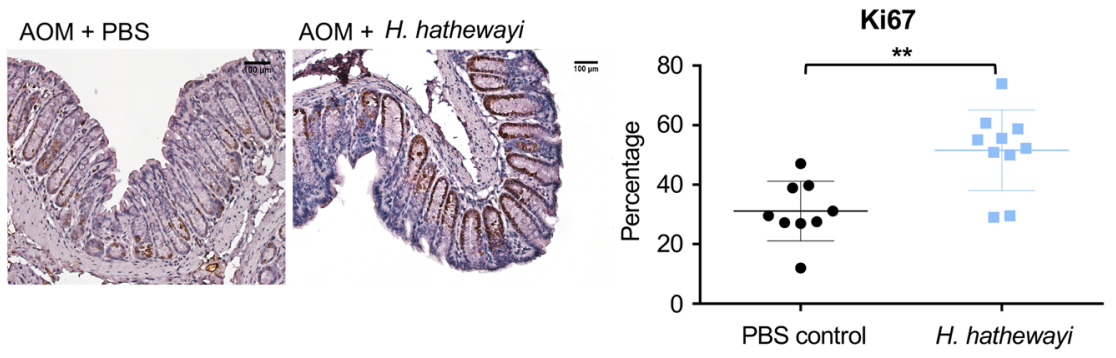

C

Germ-free mice
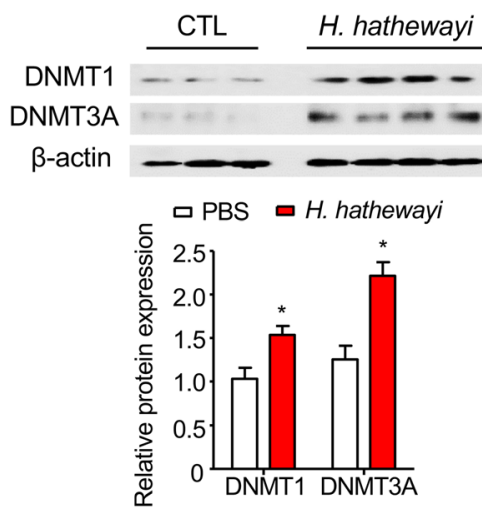

d

$$
\text { Conventional mice }
$$
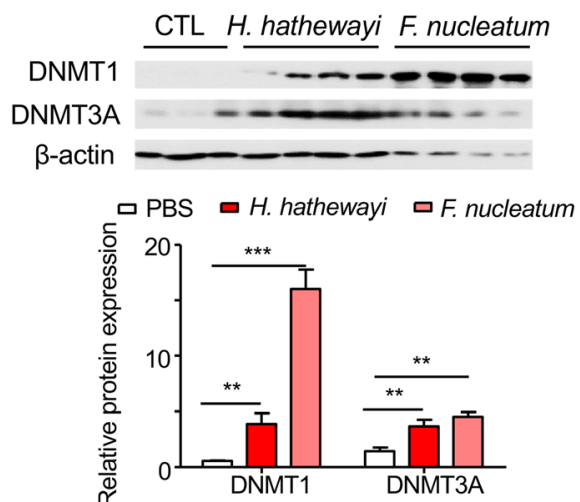

Fig. 5 Effect of $H$. hathewayi on colonic epithelial cell proliferation in germ-free and AOM-injected conventional mice. a Immunohistochemistry showing Ki-67 positive cells in the colon of germ-free mice at 20 weeks after gavaging with $\mathrm{H}$. hathewayi, and the relative proportion of Ki-67 positive cells. Scale bar: 50 m. b Immunohistochemistry showing Ki-67 positive cells in the colon of AOM-injected, microbiota-depleted convectional mice at 6 weeks after bacterial gavage, and the relative proportion of Ki-67 positive cells. Scale bar: $100 \mu$ m. C Western blots showed that $H$. hathewayi increased the colonic protein expression of DNMT1 and DNMT3A in germ-free mice. $\mathbf{d}$ Western blots showed that $F$. nucleatum and $H$. hathewayi increased colonic protein expression of DNMT1 and DNMT3A in conventional mice. Each lane in $\mathbf{c}$ and $\mathbf{d}$ corresponds to a colonic sample harvested from an individual mouse. ${ }^{*} P<0.05 ;{ }^{*} P<0.01$ significantly different between the indicated groups

abundances of CRC-enriched bacteria and pervasive hypermethylation of TSG promoters in CRC and/or colorectal adenomas. This finding is consistent with a previous study by Sobhani et al. reporting that fecal microbiota transplantation from CRC patients, as compared to healthy controls, to germ-free mice induced more hypermethylated genes in the colonic mucosa [38]. To this end, our present study complemented their work by depicting the microbiome-methylome interaction at an unprecedented resolution by assessing the epigenome-wide effect of individual bacterial species and by pinpointing $F$. nucleatum and $H$. hathewayi as two key CRC-enriched bacteria mediating promoter hypermethylation.
Previous shotgun metagenomic studies from our group and other investigators have identified $F$. nucleatum and $H$. hathewayi as two of the most significantly enriched bacteria in stools and tissue biopsies of patients with CRC $[7,39]$. F. nucleatum has already been identified as a driver bacterium to promote intestinal tumorigenesis through multiple mechanisms [19], including stimulation of the oncogenic $\mathrm{Wnt} / \beta$-catenin signaling [9]. H. hathewayi, previously known as Clostridium hathewayi, is an anaerobic, endospore-forming Gramnegative bacillus that was first described in 2001 [40, 41]. Recent studies revealed that the mucosal abundance of $H$. hathewayi was positively correlated with the 
number of $\mathrm{T}$ helper 17 cells in the intestine [42] and was associated with the disease severity of Crohn's disease [43]. Here, we for the first time characterized $H$. hathewayi as a potential CRC-promoting bacteria capable of increasing colonic epithelial cell proliferation in both conventional and germ-free mice. Nevertheless, it is noteworthy that colonic epithelial cell proliferation instead of the number of macroscopic tumors was used as a marker of cancer promotion in this study and our previous report [11]. We measured the histological outcomes at week 6 when macroscopic tumors had not yet formed in the AOM-injected conventional mouse model because we would like to assess the direct effect of $H$. hathewayi, which could not be done without effective depletion of the pre-existing gut microbiota. Since the microbiota-depleting effect of antibiotics could only last for weeks, outcomes at later time points were not measured to avoid the confounding effect of $H$. hathewayi on the gut microbiota composition. Future studies should therefore involve the use of carcinogen- or genetically-induced CRC in germ-free mice for a more comprehensive assessment of the pro-tumorigenic effect of $H$. hathewayi.

In mammals, there are three major DNMTsDNMT1, DNMT3a, and DNMT3b. DNMT1 is responsible for maintenance DNA methylation activity needed for copying the methylation pattern during DNA replication, whereas DNMT3a and 3b mediate de novo DNA methylation which is essential for genome regulation [44]. In this study, F. nucleatum and $H$. hathewayi significantly increased the global DNA methylation (5-mC) levels in colonic epithelial cell lines NCM460, HCT116, and HT29. In keeping with this, F. nucleatum and $H$. hathewayi induced the expression and nuclear activity of DNMT1 and DNMT3A in two CRC cell lines (HT29 and HCT116). A consistent upregulation of DNMT1 and DNMT3a was confirmed in mouse colonic epithelium exposed to $H$. hathewayi. To this end, both DNMT1 and DNMT3a have been shown to be essential for the tumor-initiating ability of CRC stem-like cells $[45,46]$. In a study on ulcerative colitis, a strong link between Fusobacterium enrichment and DNA methylation accumulation in the inflammatory colonic mucosa has been reported [47]. The mechanisms by which $F$. nucleatum and $H$. hathewayi promoted DNMT expression and subsequently triggered TSG promoter hypermethylation are presently unclear but higher expression of cyclooxygenase-2 (COX-2) and increased production of its product prostaglandin $\mathrm{E}_{2}$ might be involved. Moreover, upregulation of COX-2 has been documented in colonic tumors from mice inoculated with $F$. nucleatum [48], in which prostaglandin $E_{2}$ could silence TSGs via DNMT-dependent DNA hypermethylation [49]. Consistently, we observed significant upregulation of two genes involved in prostaglandin $\mathrm{E}_{2}$ production, namely Pla2g4a (encoding phospholipase A2) and Ptgs2 (encoding COX2 ), in the colonic epithelium of mice gavaged with $H$. hathewayi (Figure S4). These findings suggested that $F$. nucleatum and $H$. hathewayi might epigenetically silence TSGs in a DNMT-dependent manner via the proinflammatory $\mathrm{COX}-2 /$ prostaglandin $\mathrm{E}_{2}$ pathway. Aside from COX-2/prostaglandin $\mathrm{E}_{2}$, aberrant promoter hypermethylation of TSGs has been linked to other proinflammatory mediators. For instance, interleukin (IL)$1 \beta$ directly induced promoter methylation of $\mathrm{CDH} 1 \mathrm{en-}$ coding the tumor suppressor E-cadherin in a mouse model of gastric cancer [50]. IL-6 also induced promoter hypermethylation of several important TSGs, namely CHFR, GATA5, and PAX6, in oral cancer cells [51]. Moreover, IL-8 was shown to increase the methylation of $\mathrm{CDH1}$ gene promoter in nasopharyngeal carcinoma cells by upregulating DNMT1 via the AKT pathway [52]. These studies collectively suggested that increased levels of pro-inflammatory cytokines could drive aberrant promoter hypermethylation of TSGs.

$A P C$ promoter hypermethylation is an early event in the classical adenoma-carcinoma sequence of the colon [53]. Gene-based analysis focusing on APC demonstrated that the abundances of a number of CRCenriched bacteria, including $H$. hathewayi, F. nucleatum, and Streptococcus spp., exhibited positive correlation with $A P C$ promoter hypermethylation, suggesting that altered microbiota composition might contribute to the silencing of this prominent TSG. The loss of expression of $C D X 2$ which encodes a homeobox protein that maintains the intestinal phenotype is another common event in CRC and foreshadows poor clinical outcome [54]. In this study, the promoter hypermethylation of $C D X 2$ is strongly associated with $H$. hathewayi. Whether the mucosal abundance of $H$. hathewayi could have a similar prognostic significance as per $F$. nucleatum, however, warrants further study. EYA4 is another TSG co-regulated by $F$. nucleatum and $H$. hathewayi. Aberrant silencing of EYA4 has been shown to enhance the $\mathrm{Wnt} / \beta$-catenin signaling during CRC development [55]. Collectively, our findings suggested that altered microbiota pathogens might contribute to the silencing of the prominent TSGs through inducing promoter hypermethylation. A notable finding of this study is that $F$. nucleatum and $H$. hathewayi were associated with promoter hypermethylation of different sets of TSGs. In this regard, it is known that the direct interaction with transcription factors dictates the genomic specificity of DNMT3a and DNMT3b [56]. It is therefore possible that $F$. nucleatum and $H$. hathewayi could invoke different sets of transcription factors to mediate respective targeted DNA methylation. 


\section{Conclusions}

We demonstrated for the first time that CRC-enriched bacteria, $F$. nucleatum and $H$. hathewayi, showed strong and pervasive correlations with TSG promoter hypermethylation in clinical specimens. These bacteria could directly enhance DNA methyltransferase activity in cell-line and/or animal models. Taken together, our comprehensive integrative analysis revealed the unexpected host epigenetic regulation of multiple TSG promoters by $F$. nucleatum and $H$. hathewayi in microbiota-driven intestinal tumorigenesis.

\section{Supplementary information}

Supplementary information accompanies this paper at https://doi.org/10. 1186/s40168-020-00847-4.

Additional file 1: Figure S1. Differential genome-wide coverage of MBDCap sequence reads. Data was arcsine-square-root-transformed to help visualize low coverage regions. Rows and columns represent chromosomal segments and sample methylation profiles, respectively. Row heights are normalized relative chromosome-wide CpG density. Boxes highlight significant tissue phenotypes that had the highest mean sequence coverage for a genomic feature within a chromosome. TPM, transcripts per million. Circles, $P<0.05$; Stars, $P<0.01$. P-values were from Kruskal-Wallis tests. Figure S2. Differentially methylated regions (DMRs) in colorectal carcinogenesis. Of the 1,059 representative DMRs identified, 465 (43.9\%) were annotated with HGNCs; this subset of segments was assigned to promoter regions ( 96 or $20.6 \%$ ) in which CpG islands were present (48 or 50\%). Rows and columns represent genomic segments and sample DMR profiles, respectively. Figure S3. Heatmap of bacteria abundances in phenotype groups. From left to right the bacteria abundances are displayed in groups of normal, adenoma adjacent (AN), adenoma, CRC AN, and CRC. From top to bottom the bacteria are ranked by the fold-change between CRC and CRC AN samples. The bacteria shown are the ones with differential abundance between any two phenotype groups $(P<0.2)$. Figure S4. Induction of prostaglandin E2 biosynthetic pathway by $\mathrm{H}$. hathewayi. Expression of Pla2g4c and Cox2 in colonic epithelium of mice gavaged with or without $\mathrm{H}$. hathewayi was quantified by RT-qPCR. Expression levels were compared using t-test. *, $P<0.05$; ${ }^{*} P<$ 0.01 significantly different between the indicated groups. Supplementary Table 1. Top bacteria-methylation interactions of $C$. hathewayi and F. nuleatum with tumor suppressor genes. Supplementary Table 2. The positive significant (ZINB p-value $\leq 0.05$ ) interactions of $\mathrm{MLH} 1, \mathrm{APC}$, PTEN, P16, CDX1 and CDX2 with bacteria.

\section{Abbreviations}

5-mC: 5-Methylcytosine; COX-2: Cyclooxygenase-2; CRC: Colorectal cancer DNMT: DNA methyltransferase; MBDCap-Seq: Methyl-CpG binding domainbased capture sequencing; TSG: Tumor-suppressor gene

\section{Acknowledgements}

Not Applicable.

\section{Authors' contributions}

XX performed the statistical analysis and helped to draft the manuscript; WKKW, SHW, and MHW designed and coordinated the study and drafted the manuscript. DL, TNYK, and LZ performed cell-line and animal experiments; GN and OOC performed bioinformatic analysis; PSY, YMC, and MWYC performed methyl-CpG binding domain-based capture sequencing; ZC, YKY, XW, WYC, MTVC, PKSC, and JJYS coordinated the study; JY conceived of the study, and participated in its design and coordination and helped to draft the manuscript. All authors read and approved the final manuscript.

\section{Funding}

This project was supported by Science and Technology Program Grant Shenzhen (JCYJ20170413161534162), HMRF Hong Kong (17160862), RGC-CRF
Hong Kong (C4039-19G), RGC-GRF Hong Kong (14163817), Vice-Chancellor's Discretionary Fund CUHK and CUHK direct grant, Shenzhen Virtual University Park Support Scheme to CUHK Shenzhen Research Institute, and National Natural Science Foundation of China Grant (31871340).

\section{Availability of data and materials}

Complete datasets supporting the findings of this article are in the European Bioinformatics Institute (EBI)-European Nucleotide Archive (accession number: PRJEB35776)

\section{Ethics approval and consent to participate}

All subjects had given written informed consent. The clinical study protocol was approved by The Joint Chinese University of Hong Kong-New Territories East Cluster Clinical Research Ethics Committee. All animal studies were performed in accordance with guidelines approved by the Animal Experimentation Ethics Committee of The Chinese University of Hong Kong.

Consent for publication

Not applicable.

\section{Competing interests}

The authors declare that they have no competing interests.

\section{Author details}

${ }^{1}$ Division of Biostatistics, Centre for Clinical Research and Biostatistics, JC School of Public Health and Primary Care, The Chinese University of Hong Kong, Shatin, N.T., Hong Kong, Special Administrative Region of China. ${ }^{2}$ Department of Anaesthesia and Intensive Care, The Chinese University of Hong Kong, Shatin, N.T., Hong Kong, Special Administrative Region of China. ${ }^{3} \mathrm{Li}$ Ka Shing Institute of Health Sciences, The Chinese University of Hong Kong, Shatin, N.T., Hong Kong, Special Administrative Region of China. ${ }^{4}$ State Key Laboratory of Digestive Diseases, Institute of Digestive Diseases, CUHK-Shenzhen Research Institute, The Chinese University of Hong Kong, Shatin, N.T., Hong Kong, Special Administrative Region of China. ${ }^{5}$ Division of Hematology, Department of Internal Medicine, The Ohio State University, Columbus, OH, USA. ${ }^{6}$ Department of Biomedical Sciences, National Chung Cheng University, Chia-Yi, Taiwan, Republic of China. ${ }^{7}$ Department of Microbiology, The Chinese University of Hong Kong, Shatin, N.T., Hong Kong, Special Administrative Region of China. ${ }^{8}$ Department of Medicine and Therapeutics, The Chinese University of Hong Kong, Shatin, N.T., Hong Kong, Special Administrative Region of China.

Received: 18 November 2019 Accepted: 26 April 2020

Published online: 16 July 2020

\section{References}

1. Bray F, Ferlay J, Soerjomataram I, Siegel RL, Torre LA, Jemal A. Global cancer statistics 2018: GLOBOCAN estimates of incidence and mortality worldwide for 36 cancers in 185 countries. CA Cancer J Clin. 2018:68(6):394-424.

2. Sobhani I, Amiot A, Le Baleur Y, Levy M, Auriault ML, Van Nhieu JT, Delchier JC. Microbial dysbiosis and colon carcinogenesis: could colon cancer be considered a bacteria-related disease? Therap Adv Gastroenterol. 2013;6(3): 215-29.

3. Wong SH, Kwong TNY, Wu CY, Yu J. Clinical applications of gut microbiota in cancer biology. Semin Cancer Biol. 2019;55:28-36.

4. $\mathrm{Yu}$ J, Feng $\mathrm{Q}$, Wong $\mathrm{SH}$, Zhang $\mathrm{D}$, Liang QY, Qin Y, Tang L, Zhao $\mathrm{H}$, Stenvang J, Li Y, Wang X, Xu X, Chen N, Wu WK, Al-Aama J, Nielsen HJ, Kiilerich P, Jensen BA, Yau TO, Lan Z, Jia H, Li J, Xiao L, Lam TY, Ng SC, Cheng AS, Wong W, Chan FK, Xu X, Yang H, Madsen L, Datz C, Tilg H, Wang J, Brünner N, Kristiansen K, Arumugam M, Sung JJ, Wang J. Metagenomic analysis of faecal microbiome as a tool towards targeted non-invasive biomarkers for colorectal cancer. Gut. 2017;66(1):70-8.

5. Nakatsu G, Li X, Zhou H, Sheng J, Wong SH, Wu WK, Ng SC, Tsoi H, Dong Y, Zhang N, He Y, Kang Q, Cao L, Wang K, Zhang J, Liang Q, Yu J, Sung JJ. Gut mucosal microbiome across stages of colorectal carcinogenesis. Nat Commun. 2015;6:8727

6. Dai Z, Coker OO, Nakatsu G, Wu WKK, Zhao L, Chen Z, Chan FKL, Kristiansen $\mathrm{K}$, Sung JJY, Wong SH, Yu J. Multi-cohort analysis of colorectal cancer metagenome identified altered bacteria across populations and universal bacterial markers. Microbiome. 2018;6(1):70. 
7. Wirbel J, Pyl PT, Kartal E, Zych K, Kashani A, Milanese A, Fleck JS, Voigt AY, Palleja A, Ponnudurai R, Sunagawa S, Coelho LP, Schrotz-King P, Vogtmann E, Habermann N, Niméus E, Thomas AM, Manghi P, Gandini S, Serrano D, Mizutani S, Shiroma H, Shiba S, Shibata T, Yachida S, Yamada T, Waldron L, Naccarati A, Segata N, Sinha R, Ulrich CM, Brenner $\mathrm{H}$, Arumugam M, Bork P, Zeller G. Meta-analysis of fecal metagenomes reveals global microbial signatures that are specific for colorectal cancer. Nat Med. 2019;25(4):679-89.

8. Dalmasso G, Cougnoux A, Delmas J, Darfeuille-Michaud A, Bonnet R. The bacterial genotoxin colibactin promotes colon tumor growth by modifying the tumor microenvironment. Gut Microbes. 2014;5(5):675-80.

9. Rubinstein MR, Wang X, Liu W, Hao Y, Cai G, Han YW. Fusobacterium nucleatum promotes colorectal carcinogenesis by modulating $\mathrm{E}$-cadherin/ $\beta$ catenin signaling via its FadA adhesin. Cell Host Microbe. 2013;14(2):195-206.

10. Tsoi H, Chu ESH, Zhang X, Sheng J, Nakatsu G, Ng SC, Chan AWH, Chan FKL, Sung JJY, Yu J. Peptostreptococcus anaerobius induces intracellular cholesterol biosynthesis in colon cells to induce proliferation and causes dysplasia in mice. Gastroenterology. 2017;152(6):1419-1433.e5.

11. Wong SH, Zhao L, Zhang X, Nakatsu G, Han J, Xu W, Xiao X, Kwong TNY, Tsoi H, Wu WKK, Zeng B, Chan FKL, Sung JJY, Wei H, Yu J. Gavage of fecal samples from patients with colorectal cancer promotes intestinal carcinogenesis in germ-free and conventional mice. Gastroenterology. 2017; 153(6):1621-1633.e6.

12. Wu WK, Wang XJ, Cheng AS, Luo MX, Ng SS, To KF, Chan FK, Cho CH, Sung $\mathrm{JJ}, \mathrm{Yu} \mathrm{J}$. Dysregulation and crosstalk of cellular signaling pathways in colon carcinogenesis. Crit Rev Oncol Hematol. 2013;86(3):251-77.

13. Tse JWT, Jenkins $\sqcup$, Chionh F, Mariadason JM. Aberrant DNA Methylation in colorectal cancer: what should we target? Trends Cancer. 2017;3(10):698-712.

14. Pan X, Gong D, Nguyen DN, Zhang X, Hu Q, Lu H, Fredholm M, Sangild PT, Gao F. Early microbial colonization affects DNA methylation of genes related to intestinal immunity and metabolism in preterm pigs. DNA Res. https://doi.org/10.1093/dnares/dsy001.

15. Cortese R, Lu L, Yu Y, Ruden D, Claud EC. Epigenome-microbiome crosstalk: a potential new paradigm influencing neonatal susceptibility to disease. Epigenetics. 2016;11(3):205-15.

16. Yu DH, Gadkari M, Zhou Q, Yu S, Gao N, Guan Y, Schady D, Roshan TN, Chen MH, Laritsky E, Ge Z, Wang H, Chen R, Westwater C, Bry L, Waterland RA, Moriarty C, Hwang C, Swennes AG, Moore SR, Shen L. Postnatal epigenetic regulation of intestinal stem cells requires DNA methylation and is guided by the microbiome. Genome Biol. 2015;16:211.

17. Trimarchi MP, Murphy M, Frankhouser D, Rodriguez BA, Curfman J, Marcucci G, Yan P, Bundschuh R. Enrichment-based DNA methylation analysis using next-generation sequencing: sample exclusion, estimating changes in global methylation, and the contribution of replicate lanes. BMC Genomics. 2012;13(Suppl 8):S6.

18. Bolger AM, Lohse M, Usadel B. Trimmomatic: a flexible trimmer for Illumina sequence data. Bioinformatics. 2014;30(15):2114-20.

19. Li H, Durbin R. Fast and accurate short read alignment with BurrowsWheeler transform. Bioinformatics. 2009;25(14):1754-60

20. Morgulis A, Gertz EM, Schäffer AA, Agarwala R. A fast and symmetric DUST implementation to mask low-complexity DNA sequences. J Comput Biol. 2006;13(5):1028-40.

21. Lienhard M, Grasse S, Rolff J, Frese S, Schirmer U, Becker M, Börno S, Timmermann B, Chavez L, Sültmann H, Leschber G, Fichtner I, Schweiger MR, Herwig R. QSEA-modelling of genome-wide DNA methylation from sequencing enrichment experiments. Nucleic Acids Res. 2017;45(6):e44.

22. Heinz S, Benner C, Spann N, Bertolino E, Lin YC, Laslo P, Cheng JX, Murre C, Singh $\mathrm{H}$, Glass CK. Simple combinations of lineage-determining transcription factors prime cis-regulatory elements required for macrophage and B cell identities. Mol Cell. 2010;38(4):576-89.

23. Gusnanto A, Taylor CC, Nafisah I, Wood HM, Rabbitts P, Berri S. Estimating optimal window size for analysis of low-coverage next-generation sequence data. Bioinformatics. 2014;30(13):1823-9.

24. Benjamini Y, Speed TP. Summarizing and correcting the $\mathrm{GC}$ content bias in high-throughput sequencing. Nucleic Acids Res. 2012;40(10):e72.

25. Luo Y, Wong CJ, Kaz AM, Dzieciatkowski S, Carter KT, Morris SM, Wang J, Willis JE, Makar KW, Ulrich CM, Lutterbaugh JD, Shrubsole MJ, Zheng W, Markowitz SD, Grady WM. Differences in DNA methylation signatures reveal multiple pathways of progression from adenoma to colorectal cancer. Gastroenterology. 2014;147(2):418-29.e8.
26. Timp W, Bravo HC, McDonald OG, Goggins M, Umbricht C, Zeiger M, Feinberg AP, Irizarny RA. Large hypomethylated blocks as a universal defining epigenetic alteration in human solid tumors. Genome Med. 2014;6(8):61.

27. Aryee MJ, Jaffe AE, Corrada-Bravo H, Ladd-Acosta C, Feinberg AP, Hansen $K D$, Irizarry RA. Minfi: a flexible and comprehensive bioconductor package for the analysis of Infinium DNA methylation microarrays. Bioinformatics. 2014;30(10):1363-9.

28. Maksimovic J, Gordon L, Oshlack A. SWAN: subset-quantile within array normalization for illumina infinium HumanMethylation450 BeadChips. Genome Biol. 2012;13(6):R44.

29. Johnson WE, Li C, Rabinovic A. Adjusting batch effects in microarray expression data using empirical Bayes methods. Biostatistics. 2007;8(1):118-27.

30. Zhou W, Laird PW, Shen H. Comprehensive characterization, annotation and innovative use of Infinium DNA methylation BeadChip probes. Nucleic Acids Res. 2017:45(4):e22.

31. Abugessaisa I, Noguchi S, Hasegawa A, Harshbarger J, Kondo A, Lizio M, Severin J, Carninci P, Kawaji H, Kasukawa T. FANTOM5 CAGE profiles of human and mouse reprocessed for GRCh38 and GRCm38 genome assemblies. Sci Data. 2017:4:170107.

32. de Rie D, Abugessaisa I, Alam T, Arner E, Arner P, Ashoor H, Åström G, Babina M, Bertin N, Burroughs AM, Carlisle AJ, Daub CO, Detmar M, Deviatiiarov R, Fort A, Gebhard C, Goldowitz D, Guhl S, Ha TJ, Harshbarger J, Hasegawa A, Hashimoto K, Herlyn M, Heutink P, Hitchens K, Hon CC, Huang E, Ishizu Y, Kai C, Kasukawa T, Klinken P, Lassmann T, Lecellier CH, Lee W, Lizio M, Makeev V, Mathelier A, Medvedeva YA, Mejhert N, Mungall CJ, Noma S, Ohshima M, Okada-Hatakeyama M, Persson H, Rizzu P, Roudnicky F, Sæatrom P, Sato H, Severin J, Shin JW, Swoboda RK, Tarui H, Toyoda H, Vitting-Seerup K, Winteringham L, Yamaguchi Y, Yasuzawa K, Yoneda M, Yumoto N, Zabierowski S, Zhang PG, Wells CA, Summers KM, Kawaji H, Sandelin A, Rehli M, FANTOM Consortium, Hayashizaki Y, Carninci P, Forrest ARR, de Hoon MJL. An integrated expression atlas of miRNAs and their promoters in human and mouse. Nat Biotechnol. 2017;35(9):872-8.

33. Ritchie ME, Phipson B, Wu D, Hu Y, Law CW, Shi W, Smyth GK. limma powers differential expression analyses for RNA-sequencing and microarray studies. Nucleic Acids Res. 2015;43(7):e47.

34. Dembélé $D$, Kastner $P$. Fold change rank ordering statistics: a new method for detecting differentially expressed genes. BMC Bioinformatics. 2014;15:14.

35. Zhao M, Kim P, Mitra R, Zhao J, Zhao Z. TSGene 2.0: an updated literaturebased knowledgebase for tumor suppressor genes. Nucleic Acids Res. 2016; 44(D1):D1023-31.

36. Weiss S, Xu ZZ, Peddada S, Amir A, Bittinger K, Gonzalez A, Lozupone C, Zaneveld JR, Vázquez-Baeza Y, Birmingham A, Hyde ER, Knight R. Normalization and microbial differential abundance strategies depend upon data characteristics. Microbiome. 2017;5(1):27.

37. Holt RA, Cochrane K. Tumor potentiating mechanisms of Fusobacterium nucleatum, a multifaceted microbe. Gastroenterology. 2017;152(4):694-6.

38. Sobhani I, Bergsten E, Couffin S, Amiot A, Nebbad B, Barau C, de' Angelis N, Rabot S, Canoui-Poitrine F, Mestivier D, Pédron T, Khazaie K, Sansonetti PJ. Colorectal cancer-associated microbiota contributes to oncogenic epigenetic signatures. Proc Natl Acad Sci U S A. 2019;116(48):24285-95.

39. Liang $Q$, Chiu J, Chen $Y$, Huang $Y$, Higashimori A, Fang J, Brim H, Ashktorab H, Ng SC, Ng SSM, Zheng S, Chan FKL, Sung JJY, Yu J. Fecal bacteria act as novel biomarkers for noninvasive diagnosis of colorectal cancer. Clin Cancer Res. 2017;23(8):2061-70.

40. Steer T, Collins MD, Gibson GR, Hippe H, Lawson PA. Clostridium hathewayi sp. nov., from human faeces. Syst Appl Microbiol. 2001;24(3):353-7.

41. Kaur S, Yawar M, Kumar PA, Suresh K. Hungatella effluvii gen. nov., sp. nov., an obligately anaerobic bacterium isolated from an effluent treatment plant and reclassification of Clostridium hathewayi as Hungatella hathewayi gen. nov., comb. nov. Int J Syst Evol Microbiol. 2014;64(Pt 3):710-8.

42. Atarashi K, Tanoue T, Ando M, Kamada N, Nagano Y, Narushima S, Suda W, Imaoka A, Setoyama H, Nagamori T, Ishikawa E, Shima T, Hara T, Kado S, Jinnohara T, Ohno H, Kondo T, Toyooka K, Watanabe E, Yokoyama S, Tokoro S, Mori H, Noguchi Y, Morita H, Ivanov II, Sugiyama T, Nuñez G, Camp JG, Hattori M, Umesaki Y, Honda K. Th17 cell induction by adhesion of microbes to intestinal epithelial cells. Cell. 2015;163(2):367-80.

43. Nishino K, Imaeda H, Sakai S, Ohno M, Nishida A, Andoh A. The abundance of Clostridium hathewayi, a potent inducer of T helper 17 (Th17) cells, is associated with the disease severity of Crohn's disease. Gastroenterology. 2017:152(5 Supplement 1):S993. 
44. Lyko F. The DNA methyltransferase family: a versatile toolkit for epigenetic regulation. Nat Rev Genet. 2018;19(2):81-92.

45. Morita R, Hirohashi Y, Suzuki H, Takahashi A, Tamura Y, Kanaseki T, Asanuma H, Inoda S, Kondo T, Hashino S, Hasegawa T, Tokino T, Toyota M, Asaka M, Torigoe T, Sato N. DNA methyltransferase 1 is essential for initiation of the colon cancers. Exp Mol Pathol. 2013;94(2):322-9.

46. Weis B, Schmidt J, Maamar H, Raj A, Lin H, Tóth C, Riedmann K, Raddatz G, Seitz HK, Ho AD, Lyko F, Linhart HG. Inhibition of intestinal tumor formation by deletion of the DNA methyltransferase 3a. Oncogene. 2015;34(14):1822-30.

47. Tahara T, Hirata I, Nakano N, Tahara S, Horiguchi N, Kawamura T, Okubo M, Ishizuka T, Yamada H, Yoshida D, Ohmori T, Maeda K, Komura N, Ikuno H, Jodai Y, Kamano T, Nagasaka M, Nakagawa Y, Tuskamoto T, Urano M, Shibata T, Kuroda M, Ohmiya N. Potential link between Fusobacterium enrichment and DNA methylation accumulation in the inflammatory colonic mucosa in ulcerative colitis. Oncotarget. 2017;8(37):61917-26.

48. Kostic AD, Chun E, Robertson L, Glickman JN, Gallini CA, Michaud M, Clancy TE, Chung DC, Lochhead P, Hold GL, El-Omar EM, Brenner D, Fuchs CS, Meyerson M, Garrett WS. Fusobacterium nucleatum potentiates intestinal tumorigenesis and modulates the tumor-immune microenvironment. Cell Host Microbe. 2013;14(2):207-15.

49. Xia D, Wang D, Kim SH, Katoh H, DuBois RN. Prostaglandin E2 promotes intestinal tumor growth via DNA methylation. Nat Med. 2012;18(2):224-6.

50. Huang FY, Chan AO, Rashid A, Wong DK, Seto WK, Cho CH, Lai CL, Yuen MF. Interleukin-1 $\beta$ increases the risk of gastric cancer through induction of aberrant DNA methylation in a mouse model. Oncol Lett. 2016;11(4):2919-24.

51. Gasche JA, Hoffmann J, Boland CR, Goel A. Interleukin-6 promotes tumorigenesis by altering DNA methylation in oral cancer cells. Int J Cancer. 2011;129(5):1053-63.

52. Zhang RL, Peng LX, Yang JP, Zheng LS, Xie P, Wang MY, Huang BJ, Zhao $H R$, Bao YX, Qian CN. IL-8 suppresses E-cadherin expression in nasopharyngeal carcinoma cells by enhancing E-cadherin promoter DNA methylation. Int J Oncol. 2016;48(1):207-14.

53. Liang TJ, Wang HX, Zheng YY, Cao YQ, Wu X, Zhou X, Dong SX. APC hypermethylation for early diagnosis of colorectal cancer: a meta-analysis and literature review. Oncotarget. 2017;8(28):46468-79.

54. Graule J, Uth K, Fischer E, Centeno I, Galván JA, Eichmann M, Rau TT, Langer R, Dawson H, Nitsche U, Traeger P, Berger MD, Schnüriger B, Hädrich M, Studer P, Inderbitzin D, Lugli A, Tschan MP, Zlobec I. CDX2 in colorectal cancer is an independent prognostic factor and regulated by promoter methylation and histone deacetylation in tumors of the serrated pathway. Clin Epigenetics. 2018;10(1):120

55. Kim SJ, Tae CH, Hong SN, Min BH, Chang DK, Rhee PL, Kim JJ, Kim HC, Kim $\mathrm{DH}, \mathrm{Kim}$ YH. EYA4 acts as a new tumor suppressor gene in colorectal cancer. Mol Carcinog. 2015;54(12):1748-57.

56. Hervouet E, Vallette FM, Cartron PF. Dnmt3/transcription factor interactions as crucial players in targeted DNA methylation. Epigenetics. 2009;4(7):487-99.

\section{Publisher's Note}

Springer Nature remains neutral with regard to jurisdictional claims in published maps and institutional affiliations.

\section{Ready to submit your research? Choose BMC and benefit from:}

- fast, convenient online submission

- thorough peer review by experienced researchers in your field

- rapid publication on acceptance

- support for research data, including large and complex data types

- gold Open Access which fosters wider collaboration and increased citations

- maximum visibility for your research: over $100 \mathrm{M}$ website views per year

At $\mathrm{BMC}$, research is always in progress.

Learn more biomedcentral.com/submissions 